\title{
Individual Differences in Political Ideology and Disgust Sensitivity Affect Real-Time Spoken Language Comprehension
}

\author{
Isabell Hubert Lyall and Juhani Järvikivi* \\ Department of Linguistics, University of Alberta, Edmonton, $A B$, Canada
}

Individuals' moral views have been shown to affect their event-related potentials (ERP) response to spoken statements, and people's political ideology has been shown to guide their sentence completion behavior. Using pupillometry, we asked whether political ideology and disgust sensitivity affect online spoken language comprehension. 60 native speakers of English listened to spoken utterances while their pupil size was tracked. Some of those utterances contained grammatical errors, semantic anomalies, or socio-cultural violations, statements incongruent with existing gender stereotypes and

OPEN ACCESS

Edited by:

Tim Wharton,

University of Brighton

United Kingdom

Reviewed by:

Serhii Zasiekin,

Lesya Ukrainka East European

National University, Ukraine

Quanlei Yu,

Central China Normal University, China

${ }^{*}$ Correspondence:

Juhani Järvikivi

jarvikivi@ualberta.ca

Specialty section: This article was submitted to

Language Sciences,

a section of the journal

Frontiers in Psychology

Received: 15 June 2021 Accepted: 07 September 2021

Published: 11 October 2021

Citation:

Hubert Lyall I and Järvikivi J (2021) Individual Differences in Political Ideology and Disgust Sensitivity Affect

Real-Time Spoken Language

Comprehension.

Front. Psychol. 12:699071.

doi: 10.3389/fpsyg.2021.699071 perceived speaker identity, such as "I sometimes buy my bras at Hudson's Bay," spoken by a male speaker. An individual's disgust sensitivity is associated with the Behavioral Immune System, and may be correlated with socio-political attitudes, for example regarding out-group stigmatization. We found that more disgust-sensitive individuals showed greater pupil dilation with semantic anomalies and socio-cultural violations. However, political views differently affected the processing of the two types of violations: whereas more conservative listeners showed a greater pupil response to socio-cultural violations, more progressive listeners engaged more with semantic anomalies, but this effect appeared much later in the pupil record.

Keywords: psycholinguistics, spoken language comprehension, semantic anomalies, gender stereotypes, political ideology, disgust sensitivity, speaker identity, pupillometry

\section{INTRODUCTION}

Language comprehension is a complex process: The listener has to identify the sounds in a word, recognize the word form, retrieve information associated with this form (Cutler and Clifton, 1999; Sahin et al., 2009), and integrate the word into the rapidly unfolding context within a few hundreds of milliseconds (Hagoort and Indefrey, 2014; Levinson, 2016) in an incremental fashion (Altmann and Kamide, 1999; Sedivy et al., 1999; Kamide et al., 2003; Hagoort and Van Berkum, 2007). At the same time, a body of research has shown the importance of context, broadly understood, to real-time language comprehension. For example, an utterance at odds with factual knowledge about the state of affairs in the real world, such as "Dutch trains are white" when they are in fact yellow, triggers an N400 signature at the target word white (Hagoort et al., 2004; Hagoort and Van Berkum, 2007) in the same way as a semantically anomalous word ("Dutch trains are sour"). Further, a statement about a peanut being salted can trigger this same N400 response if embedded in a (fictional) story context that does not warrant an inanimate interpretation (Nieuwland and Van Berkum, 2006). 
Important, both speaker-related attributes, for example unfamiliar accents (Porretta et al., 2016; Grey and van Hell, 2017; Porretta and Tucker, 2019; Arnhold et al., 2020) or inferred gender (Van Berkum et al., 2008), as well as listener-based individual differences (Van den Brink et al., 2012; Hubert Lyall and Järvikivi, 2021) have been found to affect language comprehension ease. In this paper, we investigate whether two further individual difference factors, an individual's propensity to disgust as well as their political views, are associated with their language processing performance.

Disgust is an emotional response to contagious and unsafe situations, such as pathogens or bodily secretions, that is intended to protect an organism from pathogen contamination (Neuberg et al., 2011; Schaller and Neuberg, 2012). Importantly, the feeling of disgust extends beyond simple pathogen avoidance to include moral disgust or purity violations (Wagemans et al., 2018), for example triggered by bad moral character (GinerSorolla and Chapman, 2017; Molho et al., 2017), socially deviant behavior associated with out-groups, or the out-groups themselves (Schaller and Neuberg, 2012; Murray and Schaller, 2016; Dawydiak et al., 2020). Out-group members effectively end up being treated like infected in-group members (Petersen, 2017). Further, disgust is closely associated with the Behavioral Immune System, whose goal it is to direct attention to a threat of pathogen contamination, in an effort to trigger behaviors that will prevent those pathogens from entering the body (Neuberg et al., 2011; Murray and Schaller, 2016; Aarøe et al., 2017).

Commonly, disgust sensitivity is higher for women than for men, and for individuals scoring higher in the Big Five traits of Agreeableness, Conscientiousness, and Neuroticism, and lower in Openness (Druschel and Sherman, 1999). Since disgust is easily triggered not just by the presence of actual pathogens, but also by members of a perceived out-group or concepts considered immoral, thresholds for disgust have been shown to predict socio-political attitudes: People who have strong negative attitudes toward socially deviant groups tend to be disgusted more easily, and are more likely to identify as conservative (Faulkner et al., 2004; Inbar et al., 2009; Smith et al., 2011; Schaller and Neuberg, 2012; Hodson and Dhont, 2015; Murray and Schaller, 2016; Tybur et al., 2016; Aarøe et al., 2017). This link between disgust sensitivity and an individual's political ideology has been traced back to notions of purity and conformity, which, in ancestral populations, may have served the purpose of keeping novel pathogens, that the in-group would have no antibodies against, at bay (Haidt and Graham, 2007; Inbar et al., 2009). Disgust thus shapes an individual's world view, attitudes, and behavior, even down to voting behavior in political elections (Park, 2015; Shook et al., 2017, 2019; Karg et al., 2019; Stewart et al., 2020).

Thus far, there is no systematic analysis of the influence of disgust sensitivity on real-time language comprehension. However, there is some evidence that individual's moral and political views affect their language processing behavior. Van Berkum et al. (2009) showed that linguistic processing cost is not just modulated by factual states of the world, but also by a person's beliefs. They measured participants' ERP responses to statements such as "I think euthanasia is an acceptable vs. unacceptable course of action." Crucially, their participants either identified as voters of a conservative party or not. They found that words inconsistent with the participants' moral values elicited an early ERP positivity between 200 and $250 \mathrm{~ms}$ and a subsequent N400 after the onset of the clashing word ("acceptable" vs. "unacceptable," depending on the individual's party denomination).

Participants' political ideology has been also shown to affect how they understand inferred causal relations in events involving interpersonal verbs such as admire, envy, or punish. Marrville (2017) showed that participants' political views significantly predicted whether participants completed sentences fragments such as "Melissa discouraged Sean because..." by continuing with the first or the second NP (Melissa or Shawn). More precisely, Marrville (2017) showed that the completions interacted with affective properties of the verbs, namely their valence and dominance, and the participants' political ideology. For example, more conservative participants tended to have more NP1 continuations for low dominant verbs when they were also high valence (thank), but more NP2 continuations when the verb was low valence (criticize). However, this pattern was reversed for more liberal participants. Recently, Niemi et al. (2020) replicated the overall pattern for political views in a similar set-up, suggesting that individuals' moral binding values such as of loyalty, obedience to authority and purity correlated with explicit causal judgments of the NP2 in the event as more likely to have allowed-and perhaps deserved-any harmful outcomes of a particular action. Together these studies suggest that individuals' political ideology is connected to how they interpret interpersonal events and, further, that it might be associated with stronger victim-blaming tendencies in more conservative-leaning participants (see also Niemi and Young, 2016).

Relatedly, recent research suggests that language comprehension is affected by listeners' personality traits that have been linked to moral and political views and disgust sensitivity. Van den Brink et al. (2012) showed that more empathetic listeners showed a larger N400 response to (socially) unusual statements (such as a male adult saying "I cannot sleep without my teddy bear") than their less empathetic counterparts. Hubert Lyall and Järvikivi (2021) showed in a pupillometry study that listeners' Big-5 personality traits systematically modulated listeners' real-time language processing in similar scenarios. For example, less open participants showed greater pupil dilation when processing spoken utterances with morpho-syntactic errors ("He frequently have burgers for dinner"), and more introverted listeners showed greater pupil dilation in response to both semantic anomalies ("I often read heads for pleasure" vs. books) and socio-cultural clashes ("I sometimes buy my bras at Hudson's Bay," vs. "ties," spoken by a male speaker). More generally, these results suggest that listener-internal factors, such as moral and political views and personality traits, interact with information inferred about the speaker and affect ERP and pupillary responses to stimuli during real-time language processing (see also Van Berkum et al., 2008; Hanulíková et al., 2012; Porretta et al., 2016; Grey and van Hell, 2017). 
In the present study, we will investigate the extent to which individuals' disgust sensitivity predicts their language processing behavior when listening to statements that include various degrees of deviance from linguistic and social norms and stereotypes. Furthermore, we will compare the effects of individuals' disgust sensitivity and political ideology. Answers to disgust sensitivity scale might reflect the respondent's moral and political views more indirectly than answers to political ideology questionnaires which can be subject to conscious manipulation due to the somewhat obvious nature of the questions.

We used pupillometry to investigate the processing of sentences containing socio-cultural clashes, semantic anomalies, and morpho-syntactic errors. Pupil size is considered an indicator of autonomous nervous system activity in humans (Steinhauer et al., 2004; Gingras et al., 2015). Specifically, it has been shown to reflect resource allocation (Rondeel et al., 2015) due to cognitive effort, workload, arousal, attention, or affect (Hess and Polt, 1960, 1964; Ahern, 1978; Ahern and Beatty, 1979; Just and Carpenter, 1993; Goldinger and Papesh, 2012; Gingras et al., 2015; Winn et al., 2018). For linguistic stimuli, pupil size has been shown to be responsive to orthographic errors (Thierfelder et al., 2020), intelligibility (Zekveld et al., 2010), ambiguity (Vogelzang et al., 2016; Winn et al., 2018), grammatical gender mismatches and semantic anomalies (Demberg and Sayeed, 2016), accentedness (Porretta and Tucker, 2019), complexity (Ahern, 1978; Ben-Nun, 1986; Just and Carpenter, 1993; Engelhardt et al., 2010), and individual difference factors (Ahern, 1978; Ahern and Beatty, 1979; Lõo et al., 2016; Hubert and Järvikivi, 2019). Importantly, the pupillometry paradigm does not require participants to complete an overt task or action, reducing the possibility of task effects.

We tracked the participants' pupil size as they listened to anomalous or unusual statements, and correlated changes in pupil size with participants' conservatism and disgust sensitivity scores. We were especially interested in pupillary changes in response to socio-cultural violations, statements that are at odds with common gender stereotypes. Stereotypes are cognitive shortcuts (Hilton and von Hippel, 1996) that are considered part of world knowledge (Carreiras et al., 1996) and activated immediately during language comprehension (Banaji and Hardin, 1996; Osterhout et al., 1997; Pyykkönen et al., 2010; Hanulíková and Carreiras, 2015; Molinaro et al., 2016). Importantly, the extent to which individuals engage in stereotyping depends on aspects of their identity (Sibley and Duckitt, 2008; Akrami et al., 2011; Quadflieg and Macrae, 2011).

In our study, socio-cultural violations rely on vocal gender, that is, the speaker's gender as inferred from their voice. Voices with lower formant frequencies, a lower fundamental frequency, and greater resonance are typically interpreted as male (Strand, 1999; Ko et al., 2006). Vocal gender has recently been found to affect the comprehension of segments containing stereotypically male or female occupations (Grant et al., 2020). We expected more disgust-sensitive individuals to allocate more resources, as indicated by a larger pupil size, to anomalous stimuli; specifically, we expected higher disgust sensitivity and conservatism scores to be correlated with a stronger response to socio-cultural clashes, due to a stronger tendency to stigmatize.

\section{METHODS}

\subsection{Participants}

82 participants completed the main experiment and post-tests for this study, which received research ethics committee approval from Research Ethics Board 2 at the University of Alberta. While non-native speakers of English were allowed to participate in the study, their data $(n=14)$ was not used in the analyses reported below. Data from participants whose comprehension question accuracy was below $80 \%(n=8)$ were removed, as attention to and comprehension of the experiment stimuli could not be guaranteed in those cases $(\min =75 \%$; $\max =100 \%$; mean $=93: 7 \%$, 95\% CI $[92.1,95.3])$. Trials during which more than $33 \%$ of data points were recorded as missing data, i.e., $\mathrm{N} / \mathrm{A}$, were excluded from analyses ( $\mathrm{n}=782 ; 8 \%$ of trials). The analyses in this paper are thus based on the data obtained from 60 native speakers of English (male/female $=8 / 52$; age $\min / \max =17 / 83$, mean $=24.9,95 \%$ CI $[21.5,28.3])$. Of those 60 participants, 38 were recruited from the University of Alberta undergraduate linguistics student pool and received course credit for their participation; the remaining 22 participants were recruited from the general population, with no restrictions on age or background, and received a small monetary compensation for their participation.

A two-tailed $t$-test showed that the age distribution differed significantly between the two recruitment strategies [mean external $_{\text {ind }}$ $=33.5,95 \%$ CI $[25.3,41.7] ;$ mean $_{\text {internal }}=19.9,95 \%$ CI $[19$, $20.8] ; t_{(21.48)}=-3.21, p=0.004$ ], with externally recruited participants being significantly older and their age distribution being much wider.

\subsection{Materials and Design}

We created 240 utterances for experimental stimuli (see Table 1; the full list of stimuli in Supplementary Materials). While the focus of our paper is on the processing of socio-cultural violations, morpho-syntactic errors and semantic anomalies were included to compare these pragmatic deviations, which violate common expectations given the context, to more languageinternal, structural and semantic-level violations. Both morphosyntactic errors and semantic anomalies have been shown to result in processing delays for reading and listening times (Just and Carpenter, 1980; Braze et al., 2002; De Vincenzi et al., 2003; Ditman et al., 2007; Tokowicz and Warren, 2010), as exemplified in augmented P600 and N400 ERP amplitudes, respectively (Kutas and Hillyard, 1980; Ni et al., 1998; Braze et al., 2002; Hagoort and Indefrey, 2014), but also in larger pupil dilation (Beatty, 1982; Engelhardt et al., 2010; Demberg and Sayeed, 2016; Zekveld et al., 2018).

All utterances followed the same syntactic pattern to ensure comparability across regions (Jegerski and VanPatten, 2013). For item recording, items were presented to one male and one female native speaker of Western Canadian English in random order, and recorded in a sound-treated booth using a Korg MR$2000 S$ studio recorder with a Countryman E6 earset microphone. Item recordings in which the prosody sounded noticeably different from those of other items were re-recorded with the speaker. Utterances were then distributed across four lists, 
TABLE 1 | Overview of stimuli and experimental conditions.

\begin{tabular}{|c|c|c|c|c|}
\hline & Clash type & \# of stimuli & Clash description & Example stimulus \\
\hline \multirow{3}{*}{$\begin{array}{l}\frac{\omega}{0} \\
\frac{2}{0} \\
0 \\
0\end{array}$} & Socio-cultural & 120 & Clash with the speaker's perceived & I usually buy my bras at Hudson's Bay, spoken by \\
\hline & Morpho-syntactic & 56 & Violation of subject-verb agreement & She usually drive her car slowly in the snow. \\
\hline & Semantic & 32 & $\begin{array}{l}\text { Semantic mismatch between the verb } \\
\text { and the object }\end{array}$ & People often read heads for pleasure at night. \\
\hline
\end{tabular}

counterbalanced for error condition (non-anomalous baseline vs. anomalous) and speaker gender (male vs. female). Each list included the same 32 filler utterances, resulting in 135 utterances per list. Each participant was presented with one list (and, accordingly, each item only once, in just one condition and spoken by one speaker).

Additionally, all items were previously rated for acceptability in a separate, off-line Likert-style ratings experiment, by a separate set of participants (see Hubert Lyall, 2019; 99 native speakers of English recruited from the pool of undergraduate linguistics students at the [University of Alberta]; male/female $=59 / 40(60 \% / 40 \%)$; age $\min / \max =17 / 31$; mean $=20.4$ years $)$. The resulting average per-item ratings were fed into the statistical models reported below as a numerical predictor.

In the main experiment, a comprehension question was presented to the participant after approximately $30 \%$ of items (i.e., each participant was presented with a question after 38 to 41 items total). Questions were 128 simple yes/no questions in line with well-established world knowledge, such as "Do giraffes have long necks?" after the unrelated filler item "Giraffes always have very long necks," to check for both attention to the experiment, and comprehension of the auditory stimuli that were presented (De Vincenzi et al., 2003; Hanulíková et al., 2012).

\subsection{Procedure}

After introducing the participants to the experimental setup, they were seated in an adjustable chair in a dimly lit experiment booth at the Centre for Comparative Psycholinguistics, University of Alberta. Light levels were kept constant throughout the experiment and for all participants. Participants were asked to place their head on a chinrest for additional stability, and to ensure a constant screen-to-eye distance. They were then instructed to follow the instructions on the screen to calibrate the eye-tracker, and to complete the experiment. During the experiment, the pupil size of the participant's right eye (cf. Kahneman and Beatty, 1966; Porretta and Tucker, 2019) was recorded at $250 \mathrm{~Hz}$ using an EyeLink 1000 system on a desktop PC.

Each trial began with a one-point drift correct, immediately followed by the display of a fixation cross at the center of the screen. Pupil size was recorded from the start of the fixation cross. Two thousand milliseconds later, the audio stimulus began to play, and pupil size was recorded until $500 \mathrm{~ms}$ after audio offset. After an inter-stimulus interval of 3,000 ms, to allow pupil dilation to return to baseline, the next trial began. Participants were given longer breaks approximately every thirty-five trials; the length of these longer breaks was up to the participant. The main experiment took between 20 and $30 \mathrm{~min}$ to complete. Participants then moved on to the post-tests described below.

\subsection{Post-questionnaires}

Participants completed three post-test questionnaires after the main experiment session, so as not to prime them toward the purpose of the study. To assess the influence of disgust sensitivity on language comprehension, the Disgust Scale Revised [DS-R; (Haidt et al., 1994) modified by Olatunji et al. (2007)], which was also used in, for example, Inbar et al., 2009, 2012; Ahn et al., 2014; Hubert and Järvikivi, 2019, was administered to participants. Participant's political views were assessed using a Wilson-Patterson-type test (Wilson and Patterson, 1968), the full version of which can be found in section 1 (Supplementary Material). This test was also chosen for results to be directly comparable to recent research involving political values and disgust sensitivity (Jost et al., 2003; Smith et al., 2011; Ahn et al., 2014; Hatemi and Verhulst, 2015). Note that the Wilson-Patterson scale is a conservatism scale; as such, high scores signify a conservative outlook. Both the political questionnaire and the DS-R were coded in E-Prime 2.0 (Psychology Software Tools Inc., 2012). In addition, data on the participants' language background, including proficiency in other languages and places the participant had lived, was collected via a pen-and-paper language background questionnaire.

Some studies have found a link between higher disgust sensitivity and a conservative world view (Graham et al., 2009; Inbar et al., 2009, 2012; Murray and Schaller, 2016; Aarøe et al., 2017); within our participant sample, we observed a weak, nonsignificant $(p>0.05)$ trend in the same direction, that is, for individuals with higher disgust sensitivity to also be more conservative ( $r=0.22, p=0.1$ ) (Supplementary Figure 1). We did not observe significant differences in the distributions of either political views or disgust sensitivity scores between men and women; however, externally recruited participants were significantly less disgust sensitive than their internally recruited peers $\left[\right.$ mean $_{\text {external }}=1.71,95 \% \mathrm{CI}[1.52,1.9] ;$ mean $_{\text {internal }}=2.16$, $95 \%$ CI $\left.[1.97,2.35] ; t_{(53.92)}=3.25, p=0.002\right]$.

\section{RESULTS}

\subsection{Data Pre-processing and Model-Fitting}

The raw pupillometry data was pre-processed in $\mathrm{R}$ (Version 3.6.3, R Core Team, 2020) and RStudio (Version 1.3.959, RStudio Team, 2020), with one data point being one pupil size sample. 
Blinks and the adjacent 20 data points (10 to the left, 10 to the right) were removed, and the onset of each target word was centered at $0 \mathrm{~ms}$. Baseline pupil sizes were calculated per participant per trial over the time period of $-200 \mathrm{~ms}$ until onset of the target word, and data points further than 2.5 SD's from the respective participant-trial baseline (3\% of total data points) were removed.

All results reported below were obtained through generalized additive mixed effects modeling (GAM modeling, or GAMM) using the mgcv (Version 1.8-28, Wood, 2011) and it sadug (Version 2.3, van Rij et al., 2017) packages in R, with relative pupil size as the dependent variable. GAM modeling is well suited to time-series data, such as pupillometry, as it is able to capture nonlinear interactions between continuous predictors without losing information in time-binning (Tremblay and Newman, 2015; van Rij et al., 2019). All models included a random smooth for participant by time, and a random intercept by item to account for individual differences within the stimuli, and for random variance between participants beyond the factors of interest. Average item rating, time ( -200 to $2,000 \mathrm{~ms}$ from target word onset), and disgust sensitivity and political ideology scores were tested as fixed predictors. Visualizations were produced using the itsadug, ggplot2 (Version 3.2.1, Wickham, 2016), and ggpubr (Version 0.3.0, Kassambara, 2020) packages. Data in the time window from $200 \mathrm{~ms}$ before target word onset to 2,000 ms after was analyzed. The models were fitted using a forwards stepwise selection procedure, where the inclusion of variables was evaluated using a combination of a $\chi^{2}$ test of REML scores via the compareML () function, visual inspection, and the estimated p-value of the smooth parameter via the report_stats () function (see e.g., Porretta and Tucker, 2019; van Rij et al., 2019).

\subsection{Morpho-Syntactic Errors}

Changes in pupil size in response to morpho-syntactic errors were modeled as a control condition (see Supplementary Table 1 for the model output). The results showed significant non-linear effects of Time, Item Rating, and and interaction between the two (all $p$ 's $<0.0001$ ). Items that contained a morpho-syntactic error were associated with significantly larger pupil dilation (Supplementary Figure 2), suggesting that the pupillometry paradigm was able to track changes in pupil size associated with a processing difficulty at an erroneous word. Individual disgust sensitivity and political views were tested as model predictors, but were not found to be associated with significant effects on pupil dilation in this condition.

\subsection{Semantic Anomalies}

The model output is depicted in Supplementary Table 2. The results showed significant non-linear effects of Time, Item Rating, and an interaction between these $(p<0.0001)$. In addition, we found a significant interaction between Disgust Sensitivity and Time ( $p$ 's $<0.0001$ ) and, importantly, a three-way non-linear interaction between Disgust Sensitivity, Time, and Item Rating ( $p$ 's $<0.0001)$. Additionally, there was a significant interaction between Political Ideology and Item rating $(p<$ 0.0001 ) that was qualified by a three-way interaction with Time ( $p$ $<0.0001$ ). In order to assess these interactions, in what follows, we will inspect the respective difference plots that depict the difference in pupil size between the anomalous/clashing and the non-anomalous/non-clashing conditions by Time from the target word onset ( $\mathrm{x}$-axis, $0-1,500 \mathrm{~ms}$ ) and participants' Political Ideology (Figure 1A) and Disgust Sensitivity (Figure 1B) scores.

Figure 1 visualizes the three-way interaction between time since onset of the target word (on the $\mathrm{x}$-axis), the respective individual difference variable (disgust sensitivity or political views on the y-axis), and clash condition (implied; see details below). The participant's pupil size is represented via a color scale: Thus, the difference in clashing vs. non-clashing conditions is implied in this type of plot; the color scale indicates the difference in pupil size when a listener encounters a clashing statement, as compared to when they encounter a non-anomalous statement. A blue color indicates a small (or even negative) change in pupil size when listening to a clashing statement (as compared to a nonanomalous statement), whereas a yellow or orange color indicates a larger dilation. We thus see that for the three-way interaction between time, the semantic anomaly condition, and political views in Figure 1A, it is more liberal listeners (lower half of the plot, $y$-axis) who experience a larger change in pupil dilation: there is a gradual change from blue to green color starting around $500 \mathrm{~ms}$ that starts turning into yellow and orange around 1,000 $\mathrm{ms}$ after onset of the target word.

As to the interaction with disgust sensitivity, visualized in Figure 1C, listeners with higher than average disgust sensitivity (upper half of $\mathrm{y}$-axis) showed a larger pupil dilation starting already around $300 \mathrm{~ms}$ after the onset of the target word.

\subsection{Socio-Cultural Violations}

Socio-cultural violations, as the main focus of analysis in this paper, are defined as statements violating common expectations regarding what a speaker of the perceived gender would be expected to say, such as an adult male saying "I cannot sleep without my teddy bear in my arms." The final model output can be found in Supplementary Table 3. The results are depicted in Figures 1B,D. Firstly, we found significant effects of Time and Item Rating, the latter suggesting that statements clashing with the speaker's perceived gender identity indeed elicited larger pupil dilation than the non-clashing ones ( $p$ 's $<0.0001$ ). Secondly, there were significant interactions between Item Rating and both the listener's political views and their disgust sensitivity ( $p$ 's $<0.0001)$ that further interacted with time ( $p$ 's $<0.0001)$. As Figure 1 shows, in both cases, it was listeners with high scores on the respective questionnaires, that is, more conservativeleaning and more disgust-sensitive listeners, respectively, who experienced a larger increase in pupil dilation in response to a socio-cultural violation.

\section{DISCUSSION}

Prior research suggests that individual's moral views (Van Berkum et al., 2009) as well as aspects of personality (Van den Brink et al., 2012; Hubert Lyall, 2019; Hubert Lyall and Järvikivi, 2021) predict online spoken language comprehension times. In this research, we asked whether and to what extent person's sensitivity to disgust and their political ideology 

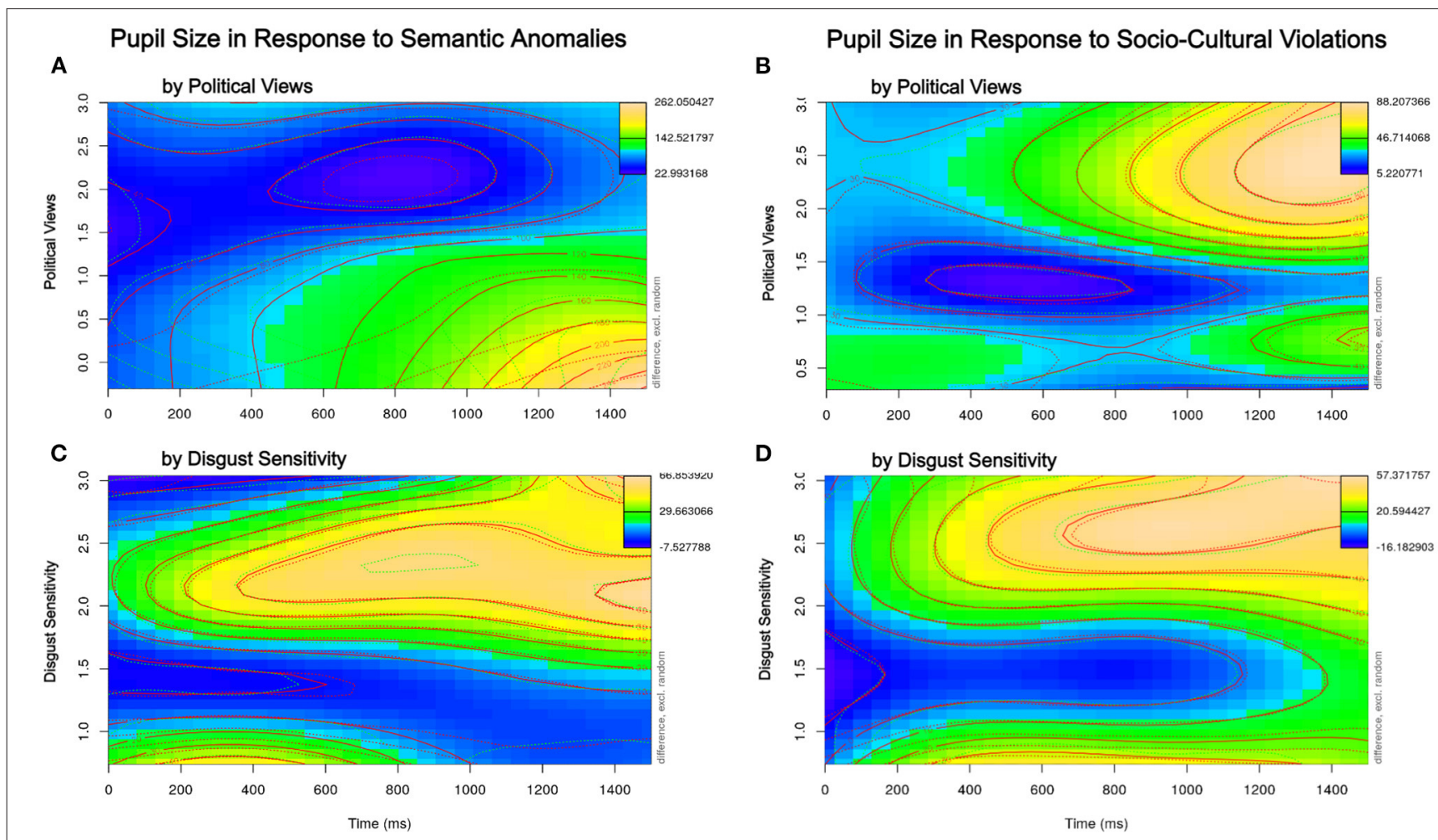

FIGURE 1 | Difference in pupil size in anomalous/clashing conditions compared to non-anomalous/non-clashing conditions by Political Views and Disgust Sensitivity ( $y$-axis) and Time from the onset of the target word (x-axis) for semantic anomalies (A,C, respectively) and socio-cultural clashes (B,D, respectively).

affect the processing of spoken utterances with different types of violations/clashes.

We found a significant effect of item rating-the extent to which a statement was grammatically, semantically, or pragmatically anomalous-for all three violation types. In all cases, listeners showed a significant pupil dilation in the clashing condition as compared to baseline, reflecting an increased processing cost as indicated by increased pupil dilation (Hess and Polt, 1960, 1964; Ahern, 1978; Ahern and Beatty, 1979; Just and Carpenter, 1993; Goldinger and Papesh, 2012; Gingras et al., 2015; Winn et al., 2018). Importantly, listeners' conservatism and disgust sensitivity scores interacted with item rating: More disgust-sensitive and conservative individuals allocated more resources to the processing of socio-cultural violations, suggesting that an individual's disgust sensitivity modulated their allocation of cognitive resources for understanding the pragmatic implications of the message. This result extends the findings from, for example, Schaller and Neuberg (2012), Murray and Schaller (2016), Aarøe et al. (2017), Wagemans et al. (2018) to the realm of real-time language comprehension, suggesting that the behavioral immune system influences an individual's response to a statement as soon as the statement is being processed.

While prior research has found that more disgust-sensitive individuals generally tend to be more conservative (Faulkner et al., 2004; Graham et al., 2009; Inbar et al., 2009, 2012; Smith et al., 2011; Schaller and Neuberg, 2012; Hodson and Dhont, 2015; Murray and Schaller, 2016; Tybur et al., 2016; Aarøe et al., 2017), the two variables were not strongly correlated in our participant sample; instead, we observed only a weak, nonsignificant correlation. This suggests that, even though the two variables may occasionally be correlated, their influence on realtime language comprehension may be distinct.

Further, with the proviso that pupillometry is not the best method to track precise processing time course, our results do suggest that the effect of the listener's political views had a later onset than that of disgust sensitivity. Thus, disgust sensitivity may affect processing earlier, perhaps affecting anticipation or immediate integration into the context.

We observed an interesting result in the processing of semantic anomalies. While more disgust-sensitive listeners experienced increased pupil dilation shortly after a semantically anomalous word, it was less conservative listeners that experienced greater resource allocation to a semantically anomalous stimulus.

The late onset of the effect in progressive listeners is in line with and could be analogous to the late posterior positivity/P600 reported in Kuperberg et al. (2020), reflecting an initial failure to integrate the anomalous utterance into the existing situation model. In this scenario, even though progressive listeners are initially not affected by the anomalous expression, they experience increased processing load at a later, integration, stage. The late posterior positivity/P600 component is also often found in response to unexpected syntactic structures, animacy violations, or semantically anomalous words, sometimes 
reflecting late, coherence establishing processes (Osterhout and Holcomb, 1992; Kuperberg et al., 2006, 2020). While the component generally peaks between 500 and $800 \mathrm{~ms}$ after stimulus onset, some prior research (see, for example, Kim and Osterhout, 2005; Nieuwland and Van Berkum, 2005) has found later peaking, around 900 to 1,000 ms after stimulus onset. Given the make-up of our stimuli, this effect would coincide timewise with the end of the experimental utterance and so with late integration.

Finally, while we found a pupil response to all three types of deviations from the norm, only the processing of semantic anomalies and socio-cultural clashes was affected by participants' political ideology and disgust sensitivity. This suggests that there may have been an emotional component to our results. Pupillometry has been shown to be sensitive to effects stemming from both increased processing load as well as from affective demands (Beatty, 1982; Steinhauer et al., 2004; Gingras et al., 2015). Research has shown that language processing is affected by mood, and happy and sad mood might engage different neural networks (Egidi and Gerrig, 2009; Egidi and Nusbaum, 2012; Egidi and Caramazza, 2014). Moreover, statements conflicting with an individual's values have been shown to quickly engage the affect system and modulate language comprehension (Van Berkum et al., 2008, 2009). Relatedly, Van Berkum et al. (2013) showed that individuals' mood affects the extent to which they engage in predictive processing during language comprehension. However, this does not seem to be the case across-the-board: they found no effect of mood for agreement violations, only for the violations of expectations as prompted by utterances depicting interpersonal events with social causes and consequences. Since both political views and disgust sensitivity have been linked to personality traits (Haidt et al., 1994; Druschel and Sherman, 1999) that themselves affect the processing of spoken statements (Hubert Lyall and Järvikivi, 2021), it may be that, at least partly, these effects stem from the same underlying emotional response (van Berkum, 2020; Wheeler et al., 2020).

In summary, our study is the first to show that an individual's disgust sensitivity, the emotional signature of the Behavioral Immune System, along with the individual's political views, modulate the resources allocated to the comprehension of an auditory linguistic message. Individuals' politically leaning toward conservatism showed more resource allocation to sociocultural violations, which aligns with prior literature suggesting that this group is more prone to elevated Behavioral Immune System activity, and out-group stigmatization (see Hodson and Dhont, 2015, for an overview). Specifically, our results are the first to show that the effects of disgust sensitivity on the comprehension of a deviating statement, via the immediate inference of vocal gender and linguistic meaning, are immediate and automatic. Our results further highlight that individual difference variables do not affect language comprehension wholesale, across the board; but that these variables interact with the type of clash being processed. The results thus support theories of language comprehension that assign importance to extra-linguistic context, consisting not only of linguistics context proper but also of individual's knowledge and conceptions of the world, and in which this extra-linguistic context influences real-time language comprehension early on. Our results further suggest that both an individual's experience with the world as well as (related) aspects of their personality may shape their real-time language processing.

\section{DATA AVAILABILITY STATEMENT}

The raw data supporting the conclusions of this article will be made available by the authors, without undue reservation.

\section{ETHICS STATEMENT}

The studies involving human participants were reviewed and approved by Research Ethics Board 2 at the University of Alberta. The patients/participants provided their written informed consent to participate in this study.

\section{AUTHOR CONTRIBUTIONS}

$\mathrm{IH}$ and $\mathrm{JJ}$ jointly conceived and designed the experiments and contributed to the writing of the manuscript. IH analyzed the data, wrote the first full draft, and performed the experiments with the assistance of undergraduate assistants. All authors contributed to the article and approved the submitted version.

\section{FUNDING}

This research was supported by a Social Sciences and Humanities Research Council of Canada (http://www.sshrc-crsh.gc.ca/) Partnership Grant (Words in the World, 895-2016-1008).

\section{ACKNOWLEDGMENTS}

We thank our undergraduate assistants Kimberly Coleman, Jessica Kondor, Sarah Noga, and Liana Oh for their assistance with experiment testing and data collection.

\section{SUPPLEMENTARY MATERIAL}

The Supplementary Material for this article can be found online at: https://www.frontiersin.org/articles/10.3389/fpsyg. 2021.699071/full\#supplementary-material

\section{REFERENCES}

Aarøe, L., Petersen, M. B., and Arceneaux, K. (2017). The behavioral immune system shapes political intuitions: why and how individual differences in disgust sensitivity underlie opposition to immigration. Am. Polit. Sci. Rev. 111, 277-294. doi: 10.1017/S0003055416000770

Ahern, S. (1978). Activation and intelligence: pupillometric correlates of individual differences in cognitive abilities 
(Ph.D. thesis). University of California, Los Angeles, CA, United States.

Ahern, S., and Beatty, J. (1979). Pupillary responses during information processing vary with scholastic aptitude test scores. Science 205, 1289-1292. doi: $10.1126 /$ science. 472746

Ahn, W. Y., Kishida, K. T., Gu, X., Lohrenz, T., Harvey, A., Alford, J. R., et al. (2014). Nonpolitical images evoke neural predictors of political ideology. Curr. Biol. 24, 2693-2699. doi: 10.1016/j.cub.2014.09.050

Akrami, N., Ekehammar, B., and Bergh, R. (2011). Generalized prejudice: common and specific components. Psychol. Sci. 22, 57-59. doi: 10.1177/0956797610390384

Altmann, G. T. M., and Kamide, Y. (1999). Incremental interpretation at verbs: restricting the domain of subsequent reference. Cognition 73, 247-264. doi: 10.1016/S0010-0277(99)00059-1

Arnhold, A., Porretta, V., Chen, A., Verstegen, S. A., Mok, I., and Järvikivi, J. (2020). (Mis) understanding your native language: regional accent impedes processing of information status. Psychon. Bull. Rev. 27, 801-808. doi: 10.3758/s13423-020-01731-w

Banaji, M. R., and Hardin, C. D. (1996). Automatic stereotyping. Psychol. Sci. 7, 136-141. doi: 10.1111/j.1467-9280.1996.tb00346.x

Beatty, J. (1982). Task-evoked pupillary responses, processing load, and the structure of processing resources. Psychol. Bull. 91, 276-292. doi: 10.1037/0033-2909.91.2.276

Ben-Nun, Y. (1986). The use of pupillometry in the study of on-line verbal processing: evidence for depths of processing. Brain Lang. 28, 1-11. doi: 10.1016/0093-934X(86)90086-6

Braze, D., Shankweiler, D., Ni, W., and Palumbo, L. C. (2002). Readers' eye movements distinguish anomalies of form and content. J. Psycholinguist. Res. 31, 25-44. doi: 10.1023/A:1014324220455

Carreiras, M., Garnham, A., Oakhill, J., and Cain, K. (1996). The use of stereotypical gender information in constructing a mental model: evidence from English and Spanish. Q. J. Exp. Psychol. 49, 639-663. doi: $10.1080 / 713755647$

Cutler, A., and Clifton, C. (1999). "Comprehending spoken language: a blueprint of the listener," in The Neurocognition of Language, Edt. Brown, C. M. and Hagoort, P. (Oxford: Oxford University Press), 123-166. doi: 10.1093/acprof:oso/9780198507932.003.0005

Dawydiak, E. J., Stafford, H. E., Stevenson, J. L., and Jones, B. C. (2020). Pathogen disgust predicts stigmatization of individuals with mental health conditions. Evol. Psychol. Sci. 6, 60-63. doi: 10.1007/s40806-019-00208-x

De Vincenzi, M., Job, R., Di Matteo, R., Angrilli, A., Penolazzi, B., Ciccarelli, L., et al. (2003). Differences in the perception and time course of syntactic and semantic violations. Brain Lang. 85, 280-296. doi: $10.1016 / \mathrm{S} 0093-934 \mathrm{X}(03) 00055-5$

Demberg, V., and Sayeed, A. (2016). The frequency of rapid pupil dilations as a measure of linguistic processing difficulty. PLOS ONE 11:e0146194. doi: 10.1371/journal.pone.0146194

Ditman, T., Holcomb, P. J., and Kuperberg, G. R. (2007). An investigation of concurrent ERP and self-paced reading methodologies. Psychophysiology 44, 927-935. doi: 10.1111/j.1469-8986.2007.00593.x

Druschel, B. A., and Sherman, M. F. (1999). Disgust sensitivity as a function of the Big Five and gender. Pers. Individ. Diff. 26, 739-748. doi: 10.1016/S0191-8869(98)00196-2

Egidi, G., and Caramazza, A. (2014). Mood-dependent integration in discourse comprehension: happy and sad moods affect consistency processing via different brain networks. Neuroimage 103, 20-32. doi: $10.1016 /$ j.neuroimage.2014.09.008

Egidi, G., and Gerrig, R. J. (2009). How valence affects language processing: negativity bias and mood congruence in narrative comprehension. Memory Cogn. 37, 547-555. doi: 10.3758/MC.37.5.547

Egidi, G., and Nusbaum, H. C. (2012). Emotional language processing: how mood affects integration processes during discourse comprehension. Brain Lang. 122, 199-210. doi: 10.1016/j.bandl.2011.12.008

Engelhardt, P. E., Ferreira, F., and Patsenko, E. G. (2010). Pupillometry reveals processing load during spoken language comprehension. Q. J. Exp. Psychol. 63, 639-645. doi: 10.1080/17470210903469864
Faulkner, J., Schaller, M., Park, J. H., and Duncan, L. A. (2004). Evolved diseaseavoidance mechanisms and contemporary xenophobic attitudes. Group Process. Intergroup Relat. 7, 333-353. doi: 10.1177/1368430204046142

Giner-Sorolla, R., and Chapman, H. A. (2017). Beyond purity: moral disgust toward bad character. Psychol. Sci. 28, 80-91. doi: 10.1177/0956797616673193

Gingras, B., Marin, M. M., Puig-Waldmuller, E., and Fitch, W. T. (2015). The eye is listening: music-induced arousal and individual differences predict pupillary responses. Front. Hum. Neurosci. 9:619. doi: 10.3389/fnhum.2015.00619

Goldinger, S. D., and Papesh, M. H. (2012). Pupil dilation reflects the creation and retrieval of memories. Curr. Direct. Psychol. Sci. 21, 90-95. doi: $10.1177 / 0963721412436811$

Graham, J., Haidt, J., and Nosek, B. A. (2009). Liberals and conservatives rely on different sets of moral foundations. J. Pers. Soc. Psychol. 96, 1029-1046. doi: $10.1037 / \mathrm{a} 0015141$

Grant, A., Grey, S., and van Hell, J. G. (2020). Male fashionistas and female football fans: gender stereotypes affect neurophysiological correlates of semantic processing during speech comprehension. J. Neurolinguist. 53, 100876-100876. doi: 10.1016/j.jneuroling.2019.100876

Grey, S., and van Hell, J. G. (2017). Foreign-accented speaker identity affects neural correlates of language comprehension. J. Neurolinguist. 42, 93-108. doi: 10.1016/j.jneuroling.2016.12.001

Hagoort, P., Hald, L., Bastiaansen, M., and Petersson, K. M. (2004). Integration of word meaning and world knowledge in language comprehension. Science 304, 438-441. doi: 10.1126/science. 1095455

Hagoort, P., and Indefrey, P. (2014). The neurobiology of language beyond single words. Annu. Rev. Neurosci. 37, 347-362. doi: 10.1146/annurev-neuro-071013-013847

Hagoort, P., and Van Berkum, J. (2007). Beyond the sentence given. Philos. Trans. R. Soc. B Biol. Sci. 362, 801-811. doi: 10.1098/rstb.2007.2089

Haidt, J., and Graham, J. (2007). When morality opposes justice: conservatives have moral intuitions that liberals may not recognize. Soc. Justice Res. 20, 98-116. doi: 10.1007/s11211-007-0034-z

Haidt, J., McCauley, C., and Rozin, P. (1994). Individual-differences in sensitivity to disgust - a scale sampling 7 domains of disgust elicitors. Pers. Individ. Diff. 16, 701-713. doi: 10.1016/0191-8869(94)90212-7

Hanulíková, A., and Carreiras, M. (2015). Electrophysiology of subjectverb agreement mediated by speakers' gender. Front. Psychol. 6:1396. doi: 10.3389/fpsyg.2015.01396

Hanulíková, A., van Alphen, P. M., van Goch, M. M., and Weber, A. (2012). When one person's mistake is another's standard usage: the effect of foreign accent on syntactic processing. J. Cogn. Neurosci. 24, 878-887. doi: 10.1162/jocn_a 00103

Hatemi, P. K., and Verhulst, B. (2015). Political attitudes develop independently of personality traits. PLoS ONE 10:e0118106. doi: 10.1371/journal.pone.0118106

Hess, E. H., and Polt, J. M. (1960). Pupil size as related to interest value of visual stimuli. Science 132, 349-350. doi: 10.1126/science.132.3423.349

Hess, E. H., and Polt, J. M. (1964). Pupil size in relation to mental activity during simple problem-solving. Science 143, 1190-1192. doi: 10.1126/science. 143.3611 .1190

Hilton, J. L., and von Hippel, W. (1996). Stereotypes. Annu. Rev. Psychol. 47, 237-271. doi: 10.1146/annurev.psych.47.1.237

Hodson, G., and Dhont, K. (2015). The person-based nature of prejudice: individual difference predictors of intergroup negativity. Eur. Rev. Soc. Psychol. 26, 1-42. doi: 10.1080/10463283.2015.1070018

Hubert Lyall, I. (2019). It's personal and disgusting: extra-linguistic information in language comprehension (Ph.D. thesis). University of Alberta, Edmonton, $\mathrm{AB}$, Canada.

Hubert Lyall, I., and Järvikivi, J. (2021). Listener's personality traits predict changes in pupil size during auditory language comprehension. Sci. Rep. 11:5443. doi: $10.1038 / \mathrm{s} 41598-021-84886-3$

Hubert, I., and Järvikivi, J. (2019). "Dark forces in language comprehension: the case of neuroticism and disgust in a pupillometry study," in Annual Meeting of the Cognitive Science Society (Montreal, QC), 450-456.

Inbar, Y., Pizarro, D., Iyer, R., and Haidt, J. (2012). Disgust sensitivity, political conservatism, and voting. Soc. Psychol. Pers. Sci. 3:537-544. doi: $10.1177 / 1948550611429024$ 
Inbar, Y., Pizarro, D. A., and Bloom, P. (2009). Conservatives are more easily disgusted than liberals. Cogn. Emot. 23, 714-725. doi: $10.1080 / 02699930802110007$

Jegerski, J., and VanPatten, B. (2013). Research Methods in Second Language Psycholinguistics. Taylor \& Francis. New York: Routledge. doi: $10.4324 / 9780203123430$

Jost, J. T., Glaser, J., Kruglanski, A. W., and Sulloway, F. J. (2003). Political conservatism as motivated social cognition. Psychol. Bull. 129, 339-375. doi: $10.1037 / 0033-2909.129 .3 .339$

Just, M. A., and Carpenter, P. A. (1980). A theory of reading: from eye fixations to comprehension. Psychol. Rev. 87, 329-354. doi: 10.1037/0033-295X.87.4.329

Just, M. A., and Carpenter, P. A. (1993). The intensity dimension of thought: pupillometric indices of sentence processing. Can. J. Exp. Psychol. 47, 310-339. doi: $10.1037 / \mathrm{h} 0078820$

Kahneman, D., and Beatty, J. (1966). Pupil diameter and load on memory. Science 154, 1583-1585. doi: 10.1126/science.154.3756.1583

Kamide, Y., Altmann, G. T. M., and Haywood, S. L. (2003). The time-course of prediction in incremental sentence processing: evidence from anticipatory eye movements. J. Mem. Lang. 49, 133-156. doi: 10.1016/S0749-596X(03) 00023-8

Karg, S. T., Wiener-Blotner, A., and Schnall, S. (2019). Disgust sensitivity is associated with heightened risk perception. J. Risk Res. 22, 627-642. doi: 10.1080/13669877.2018.1474244

Kassambara, A. (2020). ggpubr: 'ggplot2' Based Publication Ready Plots. $\mathrm{R}$ package version 0.3.0. Available online at: https://CRAN.R-project.org/ package=ggpubr.

Kim, A., and Osterhout, L. (2005). The independence of combinatory semantic processing: evidence from event-related potentials. J. Mem. Lang. 52, 205-225. doi: 10.1016/j.jml.2004.10.002

Ko, S. J., Judd, C. M., and Blair, I. V. (2006). What the voice reveals: within- and between-category stereotyping on the basis of voice. Pers. Soc. Psychol. Bull. 32, 806-819. doi: 10.1177/0146167206286627

Kuperberg, G. R., Brothers, T., and Wlotko, E. W. (2020). A tale of two positivities and the N400: distinct neural signatures are evoked by confirmed and violated predictions at different levels of representation. J. Cogn. Neurosci. 32, 12-35. doi: 10.1162/jocn_a_01465

Kuperberg, G. R., Caplan, D., Sitnikova, T., Eddy, M., and Holcomb, P. J. (2006). Neural correlates of processing syntactic, semantic, and thematic relationships in sentences. Lang. Cogn. Process. 21, 489-530. doi: $10.1080 / 01690960500094279$

Kutas, M., and Hillyard, S. A. (1980). Reading senseless sentences: brain potentials reflect semantic incongruity. Sci. Transl. Med. 207, 203-205. doi: $10.1126 /$ science. 7350657

Levinson, S. C. (2016). Turn-taking in human communication - origins and implications for language processing. Trends Cogn. Sci. 20, 6-14. doi: 10.1016/j.tics.2015.10.010

Lõo, K., van Rij, J., Jarvikivi, J., and Baayen, H. (2016). "Individual differences in pupil dilation during naming task," in Proceedings of the 38th Annual Meeting of the Cognitive Science Society, Philadelphia: Cognitive Science Society. 550-555.

Marrville, C. (2017). Gender and dominance in action: world view and emotional affect in language processing and use (Ph.D. thesis). University of Alberta, Edmonton, $\mathrm{AB}$, Canada.

Molho, C., Tybur, J. M., Guler, E., Balliet, D., and Hofmann, W. (2017). Disgust and anger relate to different aggressive responses to moral violations. Psychol. Sci. 28, 609-619. doi: 10.1177/0956797617692000

Molinaro, N., Su, J. J., and Carreiras, M. (2016). Stereotypes override grammar: Social knowledge in sentence comprehension. Brain Lang. 155-156:36-43. doi: 10.1016/j.bandl.2016.03.002

Murray, D. R., and Schaller, M. (2016). The behavioral immune system: implications for social cognition, social interaction, and social influence. Adv. Exp. Soc. Psychol. 53, 75-129. doi: 10.1016/bs.aesp.2015. 09.002

Neuberg, S. L., Kenrick, D. T., and Schaller, M. (2011). Human threat management systems: self-protection and disease avoidance. Neurosci. Biobehav. Rev. 35, 1042-1051. doi: 10.1016/j.neubiorev.2010.08.011

Ni, W., Fodor, J. D., Crain, S., and Shankweiler, D. (1998). Anomaly detection: eye movement patterns. J. Psycholinguist. Res. 27, 515-539. doi: 10.1023/A:1024996828734
Niemi, L., Hartshorne, J., Gerstenberg, T., Stanley, M., and Young, L. (2020). Moral values reveal the causality implicit in verb meaning. Cogn. Sci. 44:e12838. doi: $10.1111 / \operatorname{cogs} .12838$

Niemi, L., and Young, L. (2016). When and why we see victims as responsible: the impact of ideology on attitudes toward victims. Pers. Soc. Psychol. Bull. 42:1227-1242. doi: 10.1177/0146167216653933

Nieuwland, M. S., and Van Berkum, J. J. (2005). Testing the limits of the semantic illusion phenomenon: ERPs reveal temporary semantic change deafness in discourse comprehension. Cogn. Brain Res. 24:691-701. doi: 10.1016/j.cogbrainres.2005.04.003.

Nieuwland, M. S., and Van Berkum, J. J., a. (2006). When peanuts fall in love: N400 evidence for the power of discourse. J. Cogn. Neurosci. 18, 1098-1111. doi: 10.1162/jocn.2006.18.7.1098

Olatunji, B. O., Williams, N. L., Tolin, D. F., Abramowitz, J. S., Sawchuk, C. N., Lohr, J. M., et al. (2007). The disgust scale: item analysis, factor structure, and suggestions for refinement. Psychol. Assess. 19, 281-297. doi: 10.1037/1040-3590.19.3.281

Osterhout, L., Bersick, M., and McLaughlin, J. (1997). Brain potentials reflect violations of gender stereotypes. Memory Cogn. 25, 273-285. doi: $10.3758 / \mathrm{BF} 03211283$

Osterhout, L., and Holcomb, P. J. (1992). Event-related brain potentials elicited by syntactic anomaly. J. Mem. Lang. 31, 785-806. doi: 10.1016/0749-596X(92)90039-Z

Park, J. H. (2015). Introversion and human-contaminant disgust sensitivity predict personal space. Pers. Individ. Diff. 82, 185-187. doi: 10.1016/j.paid.2015.03.030

Petersen, M. B. (2017). Healthy out-group members are represented psychologically as infected in-group members. Psychol. Sci. 28, 1857-1863. doi: 10.1177/0956797617728270

Porretta, V., and Tucker, B. V. (2019). Eyes wide open: pupillary response to a foreign accent varying in intelligibility. Front. Commun. 4:8. doi: $10.3389 /$ fcomm.2019.00008

Porretta, V., Tucker, B. V., and Järvikivi, J. (2016). The influence of gradient foreign accentedness and listener experience on word recognition. J. Phonet. 58, 1-21. doi: 10.1016/j.wocn.2016.05.006

Psychology Software Tools, Inc. (2012). E-Prime 2.0.

Pyykkönen, P., Hyönä, J., and Van Gompel, R. P. (2010). Activating gender stereotypes during online spoken language processing: evidence from visual world eye tracking. Exp. Psychol. 57, 126-133. doi: 10.1027/1618-3169/a000016

Quadflieg, S., and Macrae, C. N. (2011). Stereotypes and stereotyping: what's the brain got to do with it? Eur. Rev. Soc. Psychol. 22, 215-273. doi: 10.1080/10463283.2011.627998

R Core Team (2020). R: A Language and Environment for Statistical Computing.

Rondeel, E. W. M., van Steenbergen, H., Holland, R. W., and van Knippenberg, A. (2015). A closer look at cognitive control: differences in resource allocation during updating, inhibition and switching as revealed by pupillometry. Front. Hum. Neurosci. 9:494. doi: 10.3389/fnhum.2015.00494

RStudio Team (2020). RStudio: Integrated Develeopment Environment for $R$.

Sahin, N. T., Pinker, S., Cash, S. S., Schomer, D., and Halgren, E. (2009). Sequential processing of lexical, grammatical, and phonological information within broca's area. Science 326, 445-449. doi: 10.1126/science. 1174481

Schaller, M., and Neuberg, S. L. (2012). Danger, Disease, and the Nature of Prejudice(s), Vol. 46. Elsevier Inc. Burlington: Academic Press. doi: 10.1016/B978-0-12-394281-4.00001-5

Sedivy, J. C. K., Tanenhaus, M., Chambers, C. G., and Carlson, G. N. (1999). Achieving incremental semantic interpretation through contextual representation. Cognition 71, 109-147. doi: 10.1016/S0010-0277(99)00025-6

Shook, N. J., Oosterhoff, B., Terrizzi, J. A., and Brady, K. M. (2017). "Dirty politics": the role of disgust sensitivity in voting. Transl. Issues Psychol. Sci. 3, 284-297. doi: $10.1037 /$ tps0000111

Shook, N. J., Thomas, R., and Ford, C. G. (2019). Testing the relation between disgust and general avoidance behavior. Pers. Individ. Diff. 150:109457. doi: 10.1016/j.paid.2019.05.063

Sibley, C. G., and Duckitt, J. (2008). Personality and prejudice: a metaanalysis and theoretical review. Pers. Soc. Psychol. Rev. 12, 248-279. doi: $10.1177 / 1088868308319226$

Smith, K. B., Oxley, D., Hibbing, M. V., Alford, J. R., and Hibbing, J. R. (2011). Disgust sensitivity and the neurophysiology of left- right political orientations. PLOS ONE 6:e25552. doi: 10.1371/journal.pone.0025552 
Steinhauer, S. R., Siegle, G. J., Condray, R., and Pless, M. (2004). Sympathetic and parasympathetic innervation of pupillary dilation during sustained processing. Int. J. Psychophysiol. 52, 77-86. doi: 10.1016/j.ijpsycho.2003.12.005

Stewart, P. A., Adams, T. G., and Senior, C. (2020). The effect of trait and state disgust on fear of god and sin. Front. Psychol. 11:51. doi: $10.3389 /$ fpsyg.2020.00051

Strand, E. A. (1999). Uncovering the role of gender stereotypes in speech perception. J. Lang. Soc. Psychol. 18, 86-100. doi: 10.1177/0261927X99018001006

Thierfelder, P., Durantin, G., and Wigglesworth, G. (2020). The effect of word predictability on phonological activation in cantonese reading: a study of eye-fixations and pupillary response. J. Psycholinguist. Res. 49, 779-801. doi: $10.1007 /$ s10936-020-09713-8

Tokowicz, N., and Warren, T. (2010). Beginning adult L2 learners' sensitivity to morphosyntactic violations: a self-paced reading study. Eur. J. Cogn. Psychol. 22, 1092-1106. doi: 10.1080/09541440903325178

Tremblay, A., and Newman, A. J. (2015). Modeling nonlinear relationships in ERP data using mixed-effects regression with R examples. Psychophysiology 52, 124-139. doi: 10.1111/psyp.12299

Tybur, J. M., Inbar, Y., Aaroe, L., Barclay, P., Barlow, F. K., de Barra, M., et al. (2016). Parasite stress and pathogen avoidance relate to distinct dimensions of political ideology across 30 nations. Proc. Natl. Acad. Sci. U.S.A. 113, 12408-12413. doi: 10.1073/pnas.1607398113

van Berkum, J. J. (2020). Inclusive affective neurolinguistics. Lang. Cogn. Neurosci. 35, 871-876. doi: 10.1080/23273798.2019.1665191

Van Berkum, J. J., De Goede, D., Van Alphen, P. M., Mulder, E. R., and Kerstholt, J. H. (2013). How robust is the language architecture? The case of mood. Front. Psychol. 4:505. doi: 10.3389/fpsyg.2013.00505

Van Berkum, J. J., Holleman, B., Nieuwland, M., Otten, M., and Murre, J. (2009). Right or wrong? Psychol. Sci. 20, 1092-1099. doi: 10.1111/j.1467-9280.2009.02411.x

Van Berkum, J. J., Van den Brink, D., Tesink, C. M. J. Y., Kos, M., and Hagoort, P. (2008). The neural integration of speaker and message. J. Cogn. Neurosci. 20, 580-591. doi: 10.1162/jocn.2008.20054

Van den Brink, D., Van Berkum, J. J. A., Bastiaansen, M. C. M., Tesink, C. M. J. Y., Kos, M., Buitelaar, J. K., et al. (2012). Empathy matters: ERP evidence for interindividual differences in social language processing. Soc. Cogn. Affect. Neurosci. 7, 173-183. doi: 10.1093/scan/nsq094

van Rij, J., Hendriks, P., van Rijn, H., Baayen, R. H., and Wood, S. N. (2019). Analyzing the time course of pupillometric data. Trends Hear. 23:233121651983248. doi: $10.1177 / 2331216519832483$

van Rij, J., Wieling, M., Baayen, R. H., and van Rijn, H. (2017). itsadug: Interpreting Time Series and Autocorrelated Data Using GAMMs.

Vogelzang, M., Hendriks, P., and van Rijn, H. (2016). Pupillary responses reflect ambiguity resolution in pronoun processing. Lang. Cogn. Neurosci. 3798, 1-10.
Wagemans, F. M. A., Brandt, M. J., and Zeelenberg, M. (2018). Disgust sensitivity is primarily associated with purity-based moral judgments. Emotion 18, 277-289. doi: $10.1037 / \mathrm{emo} 0000359$

Wheeler, N. E., Allidina, S., Long, E. U., Schneider, S. P., Haas, I. J., and Cunningham, W. A. (2020). Ideology and predictive processing: coordination, bias, and polarization in socially constrained error minimization. Current Opinion in Behavioral Sciences. 34, 192-198. doi: 10.1016/j.cobeha.2020. 05.002

Wickham, H. (2016). ggplot2: Elegant Graphics for Data Analysis. 2nd Edition. New York: Springer. doi: 10.1007/978-3-319-24 277-4

Wilson, G. D., and Patterson, J. R. (1968). A new measure of conservatism. Brit. J. Soc. Clin. Psychol. 7, 264-269. doi: 10.1111/j.2044-8260.1968.tb00568.x

Winn, M. B., Wendt, D., Koelewijn, T., and Kuchinsky, S. E. (2018). Best practices and advice for using pupillometry to measure listening effort: an introduction for those who want to get started. Trends Hear. 22:233121651880086. doi: $10.1177 / 2331216518800869$

Wood, S. N. (2011). Fast stable restricted maximum likelihood and marginal likelihood estimation of semiparametric generalized linear models. J. R. Stat. Soc. 73, 3-36. doi: 10.1111/j.1467-9868.2010.00749.x

Zekveld, A. A., Koelewijn, T., and Kramer, S. E. (2018). The pupil dilation response to auditory stimuli: current state of knowledge. Trends Hear. 22, 1-25. doi: $10.1177 / 2331216518777174$

Zekveld, A. A., Kramer, S. E., and Festen, J. M. (2010). Pupil response as an indication of effortful listening: the influence of sentence intelligibility. Ear Hear. 31, 480-490. doi: 10.1097/AUD.0b013e3181d4f251

Conflict of Interest: The authors declare that the research was conducted in the absence of any commercial or financial relationships that could be construed as a potential conflict of interest.

Publisher's Note: All claims expressed in this article are solely those of the authors and do not necessarily represent those of their affiliated organizations, or those of the publisher, the editors and the reviewers. Any product that may be evaluated in this article, or claim that may be made by its manufacturer, is not guaranteed or endorsed by the publisher.

Copyright () 2021 Hubert Lyall and Järvikivi. This is an open-access article distributed under the terms of the Creative Commons Attribution License (CC BY). The use, distribution or reproduction in other forums is permitted, provided the original author(s) and the copyright owner(s) are credited and that the original publication in this journal is cited, in accordance with accepted academic practice. No use, distribution or reproduction is permitted which does not comply with these terms. 